\title{
On the performance of bisecting K-means and PDDP *
}

\author{
Sergio M. Savaresi ${ }^{\dagger}$ and Daniel L. Boley ${ }^{\not}$
}

\section{Introduction and problem statement}

The problem this paper focuses on is the unsupervised clustering of a data-set. The dataset is given by the matrix $M=\left[x_{1}, x_{2}, \ldots, x_{N}\right] \in \mathfrak{R}^{p \times N}$, where each column of $M, x_{i} \in \mathfrak{R}^{p}$, is a single data-point. This is one of the more basic and common problems in fields like pattern analysis, data mining, document retrieval, image segmentation, decision making, etc. $([12,13])$. The specific problem we want to solve herein is the partition of $M$ into two sub-matrices (or sub-clusters) $M_{L} \in \mathfrak{R}^{p \times N_{L}}$ and $M_{R} \in \mathfrak{R}^{p \times N_{R}}, N_{L}+N_{R}=N$. This problem is known as bisecting divisive clustering.

Note that by recursively using a divisive bisecting clustering procedure, the dataset can be partitioned into any given number of clusters. Interestingly enough, the clusters so-obtained are structured as a hierarchical binary tree (or a binary taxonomy). This is the reason why the bisecting divisive approach is very attractive in many applications (e.g. in document-retrieval/indexing problems - see e.g. [17] and references cited therein).

Among the divisive clustering algorithms which have been proposed in the literature in the last two decades ([13]), in this paper we will focus on two techniques:

- the bisecting K-means algorithm;

- the Principal Direction Divisive Partitioning (PDDP) algorithm.

\footnotetext{
* First author supported by Consiglio Nazionale delle Ricerche (CNR) short-termmobility program. Second author supported by NSF grant IIS-9811229. Thanks are also due to Prof. Gene Golub of Dept. of Computer Science at Stanford, to Prof. Sergio Bittanti of Politecnico di Milano, and to Prof. Giovanna Gazzaniga of Pavia CNR Institute of Numerical Analysis.

$†$ Dipartimento di Elettronica e Informazione, Politecnico di Milano, Piazza L. da Vinci, 32, 20133, Milan, ITALY, savaresi@elet.polimi.it.

+ Department of Computer Science and Engineering, University of Minnesota, 4-192 EE/CSci, 200 Union St SE, Minneapolis, MN 55455, USA, boley@cs.umn.edu.
} 
K-means is probably the most celebrated and widely used clustering technique; hence it is the best representative of the class of iterative centroid-based divisive algorithms. On the other hand, PDDP is a recently proposed technique ([4-7]). It is representative of the non-iterative techniques based upon the Singular Value Decomposition (SVD) of a matrix built from the data-set.

The objective of this paper is twofold:

- $\quad$ compare the clustering performance of bisecting K-means and PDDP;

- analyze the dynamic behavior of the K-means iterative algorithm.

In the existing literature, both these issues have been considered only empirically. The performance of PDDP and K-means have been recently studied, and have been reported to be somehow similar, on the basis of a few application examples ([4-7]). As for K-means behavior, the main theoretical result known so far is [16], where it is shown that the K-means iterative procedure is guaranteed to converge; however, nothing is said about "where" and "how" it converges.

The main contribution of this work is to provide a simple mathematical explanation of some features of K-means and PDDP. This is done under the restrictive assumption that the data are uniformly distributed within a 2-dimensional ellipsoid. The main results here obtained can be summarized as follows:

- when the number of data-points tends to infinity, K-means and PDDP converge to the same solution;

- when the number of data-points tends to infinity, the iterative bisecting K-means algorithm is characterized by 2 stationary-points: one is an unstable equilibrium, one is a stable equilibrium point;

The paper is organized as follows: in Section 2 K-means and PDDP are concisely recalled and discussed; in Section 3 they are analyzed when the number of data-points tends to infinity, whereas in Section 4 an empirical analysis in the case of finite data sets is proposed. Some concluding remarks end the paper.

\section{Bisecting K-means and PDDP}

As already stated in the Introduction, this paper focuses on two bisecting divisive partitioning algorithms, which belong to different classes of methods: K-means is the most popular iterative centroid-based divisive algorithm; PDDP is the latest development of SVD-based partitioning techniques. The specific algorithms considered herein are now recalled and briefly commented. In such algorithms the definition of centroid will be used extensively; specifically, the centroid of $M$, say $w$, is given by

$w=\frac{1}{N} \sum_{j=1}^{N} M_{j}$,

where $M_{j}$ is the $j$-th columns of $M$. Similarly, the centroids of the sub-clusters $M_{L}$ and

$M_{R}$, say $w_{L}$ and $w_{R}$, are given by:

$w_{L}=\frac{1}{N_{L}} \sum_{j=1}^{N_{L}} M_{L, j} \quad w_{R}=\frac{1}{N_{R}} \sum_{j=1}^{N_{R}} M_{R, j}$,

where $M_{L, j}$ and $M_{R, j}$ are the $j$-th columns of $M_{L}$ and $M_{R}$, respectively. 


\section{Bisecting K-means.}

Step 1. (Initialization). Randomly select a point, say $c_{L} \in \mathfrak{R}^{p}$; then compute the centroid $w$ of $M$ (see (1)), and compute $c_{R} \in \mathfrak{R}^{p}$ as $c_{R}=w-\left(c_{L}-w\right)$.

Step 2. Divide $M=\left[x_{1}, x_{2}, \ldots, x_{N}\right]$ into two sub-clusters $M_{L}$ and $M_{R}$, according to the following rule:

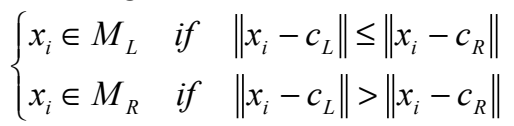

Step 3. Compute the centroids of $M_{L}$ and $M_{R}, w_{L}$ and $w_{R}$, as in (2).

Step 4. If $w_{L}=c_{L}$ and $w_{R}=c_{R}$, stop, else, let $c_{L}=w_{L}, c_{R}=w_{R}$ and go to Step 2 .

The algorithm above presented is the bisecting version of the general K-means algorithm. This bisecting algorithm has been recently discussed and emphasized in [17] and [19]. In these works it is claimed to be very effective in document-processing problems. It is here worth noting that the algorithm above recalled is the very classical and basic version of K-means, also known (see [10, 12]) as Forgy's algorithm (with a slight modification of the initialization step). Many variations of this basic version of the algorithm have been proposed, aiming to reduce the computational demand, at the price of (hopefully little) sub-optimality. Since the goal of this paper is to analyze convergence properties and clustering performance, this original version of the K-means algorithm is the most interesting and meaningful.

\section{PDDP}

Step 1. Compute the centroid w of $M$ as in (1).

Step 2. Compute the auxiliary matrix $\tilde{M}$ as $\tilde{M}=M-$ we, where e is a $N$-dimensional row vector of ones, namely $e=[1,1,1,1,1, \ldots 1]$.

Step 3. Compute the Singular Value Decompositions (SVD) of $\tilde{M}, \quad \tilde{M}=U \Sigma V^{T}$, where $\Sigma$ is a diagonal $p \times N$ matrix, and $U$ and $V$ are ortonormal unitary square matrices having dimension $p \times p$ and $N \times N$, respectively (see [11] for an exhaustive description of SVD).

Step 4. Take the first column vector of $U$, say $u=U_{1}$, and divide $M=\left[x_{1}, x_{2}, \ldots, x_{N}\right]$ into two sub-clusters $M_{L}$ and $M_{R}$, according to the following rule:

$\left\{\begin{array}{lll}x_{i} \in M_{L} & \text { if } & u^{T}\left(x_{i}-w\right) \leq 0 \\ x_{i} \in M_{R} & \text { if } & u^{T}\left(x_{i}-w\right)>0\end{array}\right.$.

The PDDP algorithm, recently proposed in [5], belongs to the class of SVD-based data-processing algorithms ([2,3]); among them, the most popular and widely known are the Latent Semantic Indexing algorithm (LSI - see [1,9]), and the LSI-related Linear Least Square Fit (LLSF) algorithm ([8]). PDDP and LSI mainly differ in the fact that the PDDP splits the matrix with hyperplane passing through its centroid; LSI through the origin. Another major feature of PDDP is that the SVD of $\tilde{M}$ (Step 3.) can be stopped at the first singular value/vector. This makes PDDP significantly less computationally demanding than LSI, especially if the data-matrix is sparse and the principal singular vector is calculated by resorting to the Lanczos technique $([11,14])$. 


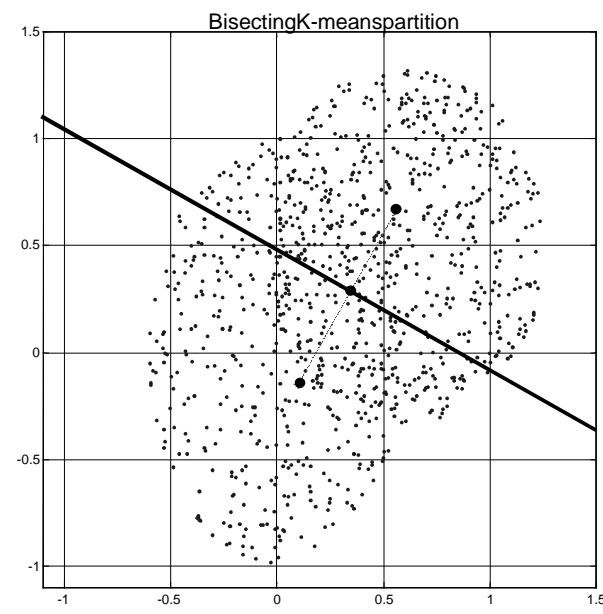

Fig. 1a. Partitioning line (bold) of bisecting K-means algorithm. The bullets are the centroids of the data-set and of the two sub-clusters.

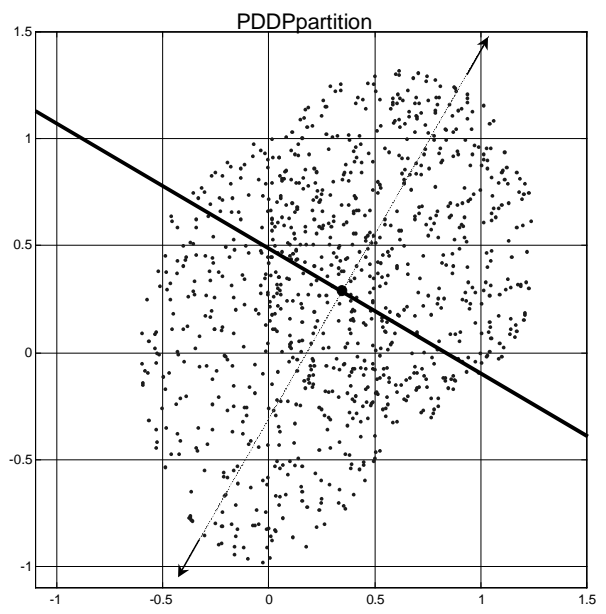

Fig.1b. Partitioning line (bold) of PDDP algorithm. The bullet is the centroid of the data set. The two arrows show the principal direction of $\tilde{M}$.

The main difference between K-means and PDDP is that K-means is based upon an iterative procedure, which, in general, provides different results for different initializations, whereas PDDP is a "one-shot" algorithm, which provides a unique solution. In order to understand better how K-means and PDDP work, in Fig.la and Fig. $1 \mathrm{~b}$ the partition of a generic matrix of dimension $2 \times 2000$ provided by K-means and PDDP, respectively, is displayed. From Fig.1, it is easy to see how K-means and PDDP work:

- the bisecting K-means algorithm splits $M$ with an hyperplane which passes through the centroid $w$ of $M$, and is perpendicular to the line passing through the centroids $w_{L}$ and $w_{R}$ of the sub-clusters $M_{L}$ and $M_{R}$. This is due to the fact that the stopping condition for K-means iterations is that each element of a cluster must be closer to the centroid of that cluster than the centroid of any other cluster.

- $\quad$ PDDP splits $M$ with an hyperplane which passes through the centroid $w$ of $M$, and is perpendicular to the principal direction of the "unbiased" matrix $\widetilde{M}$ (note that $\widetilde{M}$ is the translated version of $\mathrm{M}$, having the origin as centroid). The principal direction of $\tilde{M}$ is its direction of maximum variance (see [11]).

At a first glance, the two clusters provided by K-means and PDDP look almost indistinguishable. A more careful analysis reveals that the two partitions differ by a few points. Note that this is somewhat unexpected, since the two algorithms differ substantially.

In the rest of the paper we will try to give a rational explanation to the fact that PDDP and bisecting K-means may provide similar results. This will be done by analyzing the dynamic behavior of K-means iteration. Moreover, we will try to clearly outline the pros and cons of these two seemingly equivalent algorithms.

The analysis presented in the following two sections is based upon the restrictive assumption that the points of the data-set are uniformly distributed within an ellipsoid. This assumption deserves some comments: 
It is important pointing out that an answer to the question "where does K-means converge?" can be found only if an assumption of the data-distribution is made. Note that this is not mandatory if one only wants an answer to the question "does the K-means iteration converge?" (as a matter of fact in [16] no assumptions on the data distribution are made). Therefore, the sensible choice of the data distribution becomes the main issue.

Ellipsoid-shaped uniform distribution is the simplest distribution with compact support that, from the clustering point of view, is equivalent to multi-dimensional Gaussian distribution (which is the most typical distribution of experimental data). Henceforth it can be considered the "default" distribution when no a-priori information on the data is available.

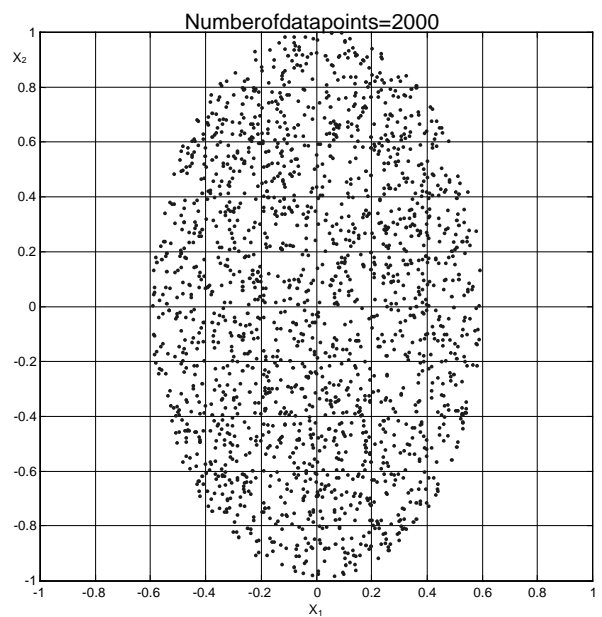

Fig.2a. 2000 data points uniformly distributed within an ellipsoid.

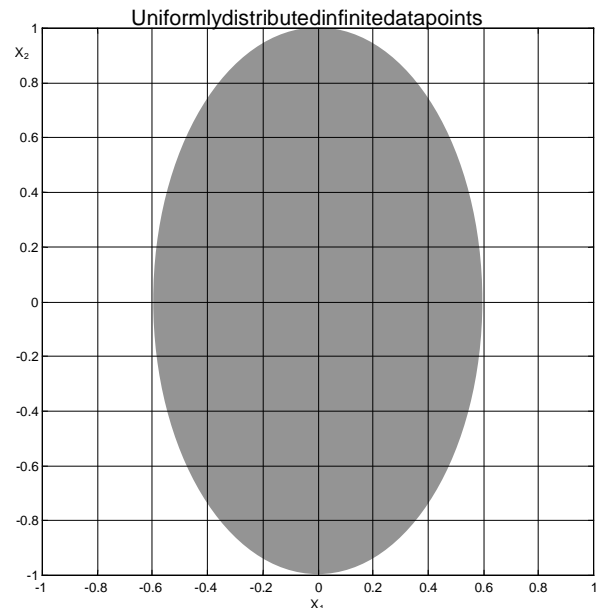

Fig.2b. Infinite data points uniformly distributed within an ellipsoid.

\section{Theoretical results for infinite data sets}

In this section the asymptotic behavior of bisecting K-means and PDDP will be analyzed. Asymptotic here means that the data set has an infinite number of points, namely $N \rightarrow \infty$. In Fig. 2 the difference between a finite and an infinite set of points is naively depicted.

We will focus on the 2-dimensional case; specifically, it is assumed that each point $x=\left[x_{1}, x_{2}\right]^{T}$ of the data-set belongs to an ellipsoid centered in the origin and referred to the axes:

$$
x=\left[x_{1}, x_{2}\right]^{T} \text { belongs to the data set if: } \quad \frac{x_{1}^{2}}{a^{2}}+x_{2}^{2} \leq 1 ;
$$

the semi-axes lengths of the ellipsoid in (3) are $a(0<a \leq 1)$ and 1 , respectively.

Given these assumptions, the problem we wish now to solve is the mathematical description of the dynamic behavior of the bisecting K-means algorithm. The solution of this problem will be given in the following four items (a)-(d).

(a) Parametrization of the splitting line. First note that the splitting hyperplane (the splitting line in 2-dimensions) is always a line passing through the origin. This property is preserved even at the first step (see the initialization procedure used in Step 1. - Section 
2). Henceforth, the splitting line can be parameterized using one parameter only. The natural choice for this parameter is the angle, say $\alpha$, between the splitting line and the positive $x_{1}$ semi-axis. We shall use the subscript " $t$ " to indicate the iteration number, namely $\alpha_{t}$ is the value of $\alpha$ at iteration $t$. With no loss of generality it is also assumed that $0 \leq \alpha_{t} \leq \pi / 2$.

(b) Description of the basic idea. The basic idea used to compute the mathematical model of the dynamic behavior of bisecting K-means is the following. Given $\alpha_{t}$, the next angle $\alpha_{t+1}$ can be calculated by first computing the centroids, say $w_{L}\left(\alpha_{t}\right)$ and $w_{R}\left(\alpha_{t}\right)$, of the two semi-clusters induced by the splitting line with angle $\alpha_{t}$. The angle $\alpha_{t+1}$ of the next-iteration splitting line then can be easily computed: it is known to be perpendicular to the line connecting $w_{L}\left(\alpha_{t}\right)$ and $w_{R}\left(\alpha_{t}\right)$. In this way we obtain a recursive relationship $\alpha_{t+1}=f\left(\alpha_{t}\right)$, which provides a complete description of the dynamic behavior of bisecting K-means.

(c) Computation of the centroids. Due to the infinite number of uniformly distributed points in the data-set, the centroids of the two sub-clusters induced by the splitting line with angle $\alpha_{t}$ must be computed using integral calculus. Using $x_{2}$ as integration variable, the computation of the position of $w_{L}$ (which is the centroid of the "Left" cluster, bordered with a dashed line in Fig.3) must be split into the computation of the centroids of two sub-pieces of the Left cluster (which are separated by the dashed-dotted line in Fig.3). The position of $w_{L}$ hence is given by:

$$
w_{L}=\left[\begin{array}{c}
w_{L 1} \\
w_{L 2}
\end{array}\right]=\left[\begin{array}{c}
\int_{-S}^{S}\left(\frac{x_{2}}{\tan \left(\alpha_{t}\right)}-a \sqrt{1-x_{2}^{2}}\right) \cdot\left(\frac{x_{2}}{\tan \left(\alpha_{t}\right)}+a \sqrt{1-x_{2}^{2}}\right) d x_{2} \\
\int_{-S}^{S}\left(\frac{x_{2}}{\tan \left(\alpha_{t}\right)}+a \sqrt{1-x_{2}^{2}}\right) d x_{2} \\
\int_{-S}^{S} x_{2} \cdot 2 a \sqrt{1-x_{2}^{2}} d x_{2} \\
\frac{\int_{-S}^{S} x_{2} \cdot\left(\frac{x_{2}}{\tan \left(\alpha_{t}\right)}+a \sqrt{1-x_{2}^{2}}\right) d x_{2}}{\int_{-S}^{S} 2 a \sqrt{1-x_{2}^{2}} d x_{2}}+\frac{x_{-S}\left(\frac{x_{2}}{\tan \left(\alpha_{t}\right)}+a \sqrt{1-x_{2}^{2}}\right) d x_{2}}{S_{-S}}
\end{array}\right],
$$

where $S$ is the $x_{2}$-coordinate of the intersection between the splitting line and the ellipsoid in the first quadrant (see Fig.3); its expression is given by:

$$
S=\frac{a \cdot \sin \left(\alpha_{t}\right)}{\sqrt{\cos ^{2}\left(\alpha_{t}\right)+a^{2} \sin ^{2}\left(\alpha_{t}\right)}} .
$$

Both (4) and (5) hold for $0<a \leq 1$ and $0 \leq \alpha_{t} \leq \pi / 2$. Fortunately, (4) can be explicitly computed and significantly simplified. After some cumbersome manipulations it can be shown that $w_{L}$ is given by:

$$
w_{L}=\left[\begin{array}{c}
w_{L 1} \\
w_{L 2}
\end{array}\right]=\left[\begin{array}{l}
-\frac{4}{3} \frac{a^{2} \sin \left(\alpha_{t}\right)}{\pi \sqrt{\cos ^{2}\left(\alpha_{t}\right)+a^{2}-a^{2} \cos ^{2}\left(\alpha_{t}\right)}} \\
\frac{4}{3} \frac{\cos \left(\alpha_{t}\right)}{\pi \sqrt{\cos ^{2}\left(\alpha_{t}\right)+a^{2}-a^{2} \cos ^{2}\left(\alpha_{t}\right)}}
\end{array}\right], 0<a \leq 1,0 \leq \alpha_{t} \leq \pi / 2 ;
$$

it is trivial to see that $w_{R}$ is given by $w_{R}=-w_{L}$. 
(d) The dynamic model of bisecting K-means. Once $w_{L}\left(\alpha_{t}\right)$ and $w_{R}\left(\alpha_{t}\right)$ have been found, it is easy to compute the recursive function $\alpha_{t+1}=f\left(\alpha_{t}\right)$ which models the transition from $\alpha_{t}$ to the angle $\alpha_{t+1}$ of the next-iteration splitting line. Indeed, this line must be perpendicular to the line passing through $w_{L}$ and $w_{R}$, namely:

$\alpha_{t+1}=\operatorname{atan}\left[a^{2} \tan \left(\alpha_{t}\right)\right], 0<a \leq 1,0 \leq \alpha_{t} \leq \pi / 2$.

Equation (6) is one of the major results of this work, since it provides a rigorous closed-form explicit expression of the dynamic behavior of bisecting K-means in the limiting case. Note that (6) represents a first order autonomous (i.e. without forcing inputs) non-linear dynamic discrete-time system. As such, it can be analyzed using nonlinear systems theory (see e.g. $[15,18]$ ). The analysis of $(6)$ reveals that:

- By solving the steady-state equation $\bar{\alpha}=\operatorname{atan}\left[a^{2} \tan (\bar{\alpha})\right]$, it is easy to see that the iterative K-means procedure can only have two stationary-points, at $\bar{\alpha}=0$ and $\bar{\alpha}=\pi / 2$. In correspondence to these points the ellipsoid is divided by its shorter axis $(\bar{\alpha}=0)$, and by its longer axis ( $\bar{\alpha}=\pi / 2)$, respectively.

- By locally linearizing the dynamic system (6) about the admissible equilibrium points (namely by computing the tangent model $\delta \alpha_{t+1}=\left(\left.\left(\partial f\left(\alpha_{t}\right) / \partial \alpha_{t}\right)\right|_{\alpha_{t}=\bar{\alpha}}\right) \delta \alpha_{t}$, where $\delta \alpha_{t}:=\alpha_{t}-\bar{\alpha}$ ), we obtain the following two linear dynamic discrete-time systems:

local dynamic behavior about $\bar{\alpha}=0: \delta \alpha_{t+1}=\left(a^{2}\right) \delta \alpha_{t}, \delta \alpha_{t}:=\alpha_{t}-0$;

local dynamic behavior about $\bar{\alpha}=\pi / 2: \delta \alpha_{t+1}=\left(1 / a^{2}\right) \delta \alpha_{t}, \delta \alpha_{t}:=\alpha_{t}-\pi / 2$.

From linear discrete-time dynamic system theory we know that, if $0<a<1$, the linear system about $\bar{\alpha}=0$ is asymptotically stable, and the linear system about $\bar{\alpha}=\pi / 2$ is unstable (indeed they have poles in $a^{2}$ and in $1 / a^{2}$, respectively). This means that bisecting K-means always converges towards $\bar{\alpha}=0$, unless the algorithm is exactly initialized with $\alpha_{0}=\pi / 2$ (namely the initial point $c_{L}$ exactly belongs to the $x_{1}$-axis). In Fig. 4 the function (6) is displayed, when $a=0.6$, and a simulated movement of system (6) is illustrated. Note that, whatever $\alpha_{0}$ is (except in the case $\left.\alpha_{0}=\pi / 2\right)$ the dynamic system $\alpha_{t+1}=f\left(\alpha_{t}\right)$ always converges in $\bar{\alpha}=0$.

- The value of $a$ strongly affects the number of iterations taken by the algorithm to converge. Thanks to equation (6) this number can be given an approximate but quantitative estimate, using dynamic systems theory. First note that the linear system described by the recursive equation $\delta \alpha_{t+1}=\left(a^{2}\right) \delta \alpha_{t}$ only asymptotically converges at its equilibrium point. A measure of the "speed" at which the system converges towards the equilibrium is given by the so-called time-constant $\tau$. $\tau$ is defined as the number of steps that $\delta \alpha_{t}$ takes to decrease its distance from 0 by a factor $1 / e$, and it is related to a by the following relationship: $\tau=\left(-\frac{1}{\log \left(a^{2}\right)}\right)$.

Due to the discrete nature of the distribution, the bisecting K-means algorithm converges in a finite number of steps, say $T . T$ is a function of the number of the 
data-points $N$ (namely it depends on how densely the data are distributed), which is expected to be proportional to $\tau$, namely:

$$
T=\gamma(N) \cdot\left(-\frac{1}{\log \left(a^{2}\right)}\right) .
$$

The exact value of $\gamma(N)$ is hard to be predicted exactly. A rule-of-thumb typically used by the control systems practitioner can be used to have an idea of $\gamma(N)$ : this rule says that, when $\delta \alpha_{t}$ has reached the $98 \%$ of the distance between the initial value and the equilibrium, the system can be considered, in practice, at steady-state. It is easy to see that this corresponds to $\gamma(N) \approx 4$. In Section 4 a numerical validation of this formula will be provided.

Finally note that $T$ may take very different values. For instance (if $\gamma(N) \approx 4$ ), Kmeans is expected to take only $10-15$ iterations to converge if $a=0.7$, about 40 iterations are needed if $a=0.9$, whereas if $a=0.95$ the algorithm might need 80 iterations to converge. It is important to observe, however, that (7) is expected to provide a reliable estimate of $T$ only if the number of the points of the data-set is large. For small data-sets the number of iterations required by K-means can be considerably smaller than (7).

The analysis above presented is the main contribution of this Section. It can be concisely summarized with the following two propositions.

Proposition 1. If the data-points of a data-set are uniformly distributed in a 2dimensional ellipsoid, the semi-axes of the hyper-ellipsoid have lengths equal to 1 and $a$, $(0<a<1)$, and $N \rightarrow \infty$, then the dynamic discrete-time system which models the K-means iterative algorithm is characterized by 2 equilibrium points; one is locally unstable, and one is locally stable. In particular, the dynamic model has the form: $\alpha_{t+1}=\operatorname{atan}\left(a^{2} \tan \left(\alpha_{t}\right)\right), 0 \leq \alpha_{t} \leq \pi / 2$. The splitting hyperplanes corresponding to the equilibrium points pass through the origin and are orthogonal to the main axes of the ellipsoid. The splitting hyperplane corresponding to the stable equilibrium point is orthogonal to the largest axis of the ellipsoid.

Proof. The proof of this result is given in items (a)-(d) above.

Proposition 2. If the data-points of a data-set are uniformly distributed in a 2dimensional ellipsoid, the semi-axes of the hyper-ellipsoid have lengths equal to 1 and $a$, $(0<a<1)$, and $N \rightarrow \infty$, then the PDDP algorithm splits the ellipsoid with an hyperplane passing through the origin and orthogonal to the largest axis of the ellipsoid.

Proof. This result is a direct implication of the properties of the SVD. Indeed the 2 singular vectors of a set of points uniformly distributed within an ellipsoid coincide with the direction of the principal axes of the ellipsoid (see [11] for details).

Propositions 1 and 2 show that bisecting K-means and PDDP provide the same solution, except in the case when the initialization of K-means exactly corresponds to an unstable equilibrium point of the K-means dynamic model. However, if the initialization is made randomly, this event occurs with probability zero.

These asymptotic results are useful to gain a deep insight into the bisecting Kmeans algorithm, and to explain why, in many cases, K-means and PDDP show a very similar clustering behavior. However, when the data set contains a finite number of data 
(namely when the number of points is comparatively small), bisecting K-means and PDDP might provide solutions, which, sometimes, are remarkably different. The finite data-set case will be analyzed and discussed in the next section, on the basis of numerical results obtained by simulation.

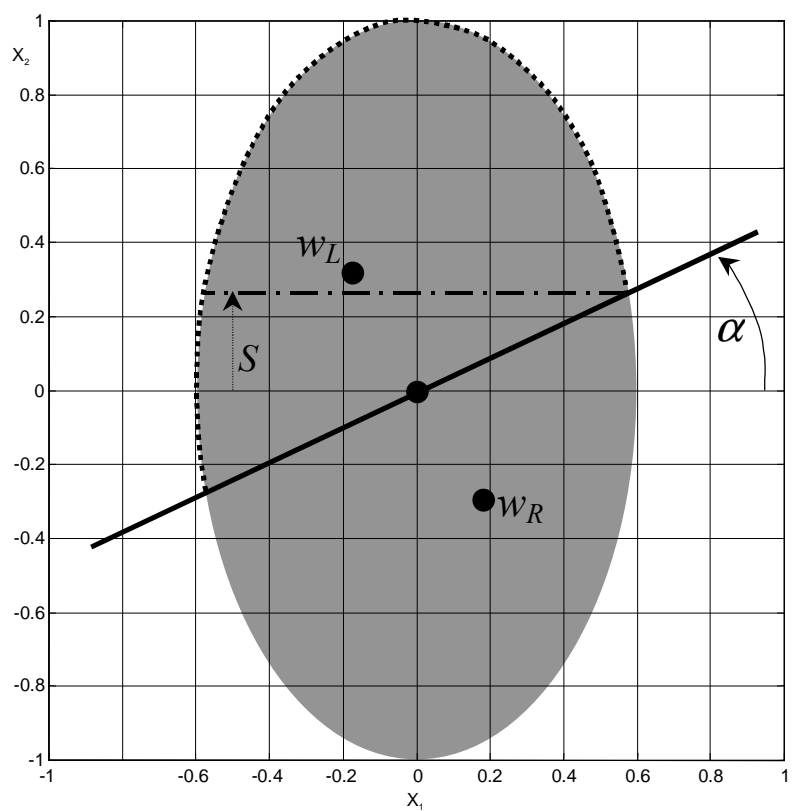

Fig.3. Parametrization of the splitting-line in K-means

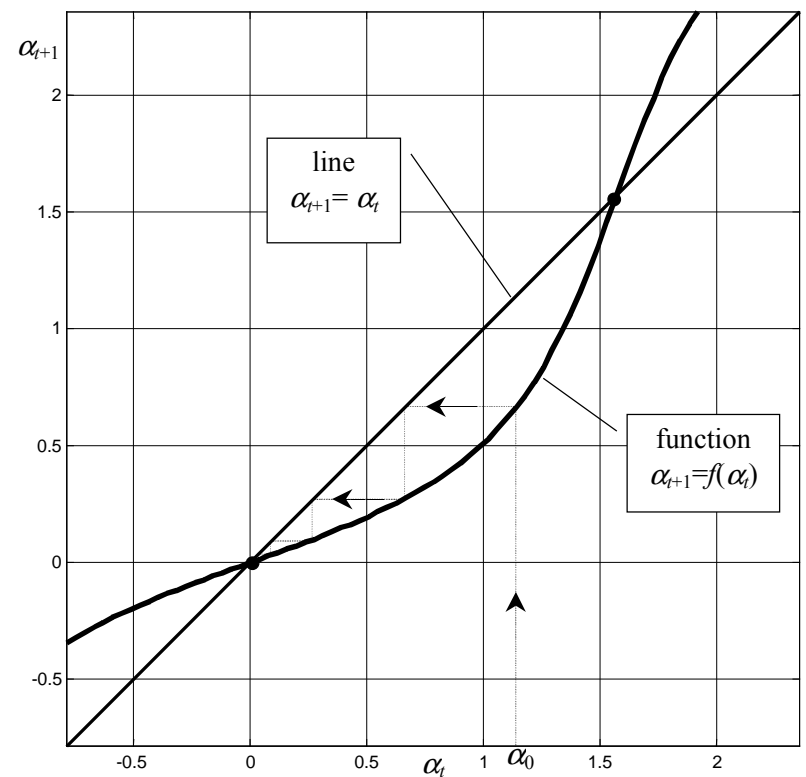

Fig.4. Function (6) (extended over the range $[-\pi / 4 ; 3 \pi / 4]$ ) when $a=0.6$. The bullets are the equilibria. The thin line is a simulated movement of (6) 


\section{Numerical results for finite data sets}

In this section, the bisecting K-means and PDDP will be analyzed when the dataset has a finite number of data-points. The analysis will be done empirically, using simulated data.

The purpose of this section is twofold:

- validate the theoretical results obtained in the previous section, and see how they change when the data-set is finite;

- $\quad$ understand the pros and cons of K-means and PDDP.

The analysis is structured as follows: first the dynamic model of K-means will be numerically computed for finite data-sets, and the problem of local minima will be discussed; then the formula (7) for the estimation of the number of iterations required by $\mathrm{K}-\mathrm{means}$ to converge will be validated.
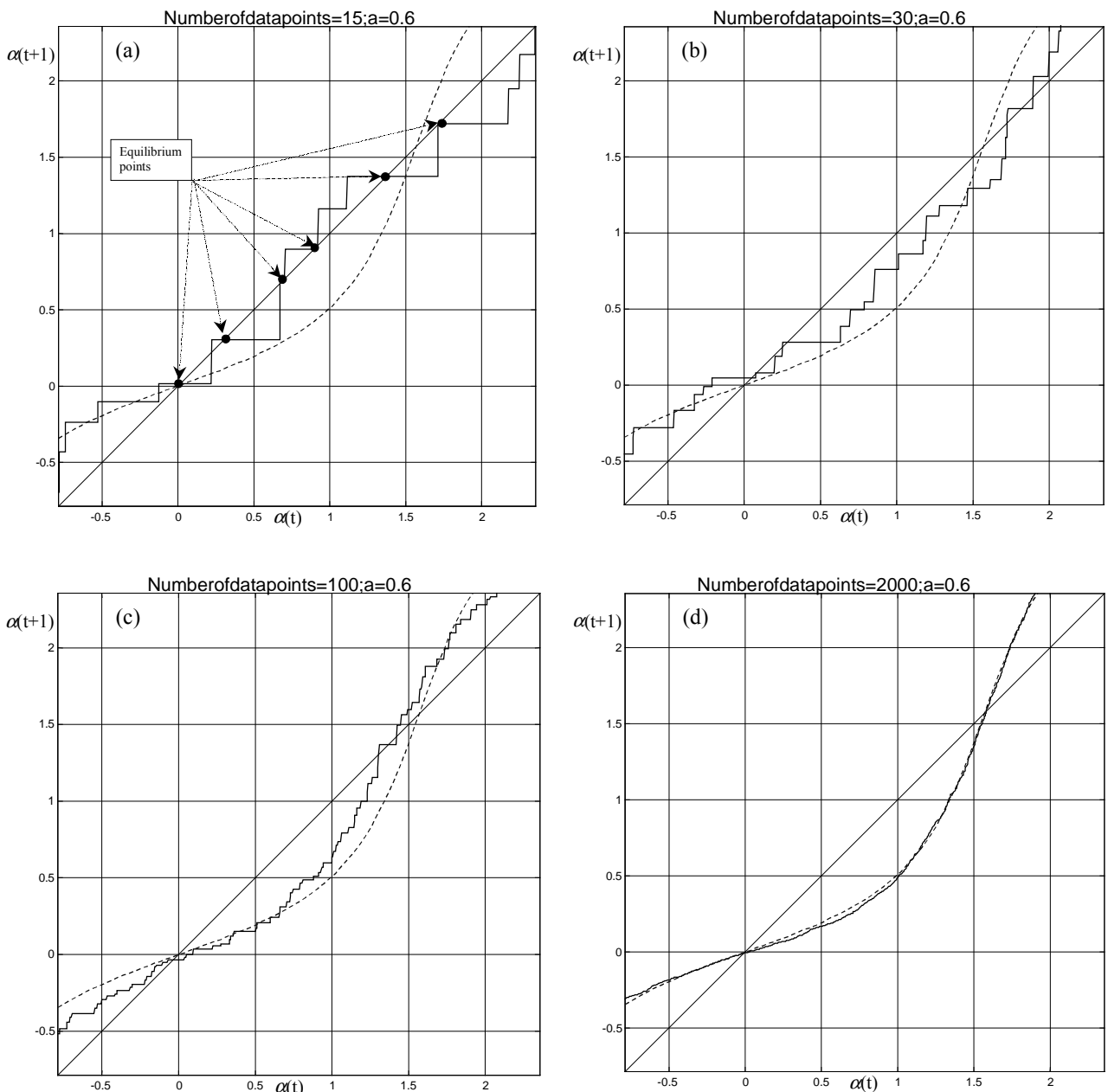

Fig.5. Recursive function $\alpha_{t+1}=f\left(\alpha_{t}\right)$ estimated from data, when $a=0.6$. The dashed line is the asymptotic function (6) computed in Section 4. (a): $N=15$; (b): $N=30$; (c): $N=100$; (d): $N=2000$. 
The first problem we consider is the analysis of the K-means dynamic behavior when the data-set has a finite number of data. As a first experiment, four sets of data have been considered, characterized by 15, 30, 100 and 2000 data-points uniformly distributed within a 2-dimensional ellipsoid with $a=0.6$. The recursive function $\alpha_{t+1}=f\left(\alpha_{t}\right)$ has been numerically computed for these four data-sets. The results are displayed in Fig.5. From the inspection of Fig.5, the following remarks can be done:

The main difference between the asymptotic function (6) and the recursive functions corresponding to finite data-sets is that the latter are step-wise functions. A major consequence of this function being step-like is that every equilibrium point (namely every point where the function crosses the line $\alpha_{t+1}=\alpha_{t}-$ see Fig.5a) is locally asymptotically stable, since the local slope of the function about the equilibrium is smaller than 1 . Note that this explains why K-means is affected by "local minima" problems.
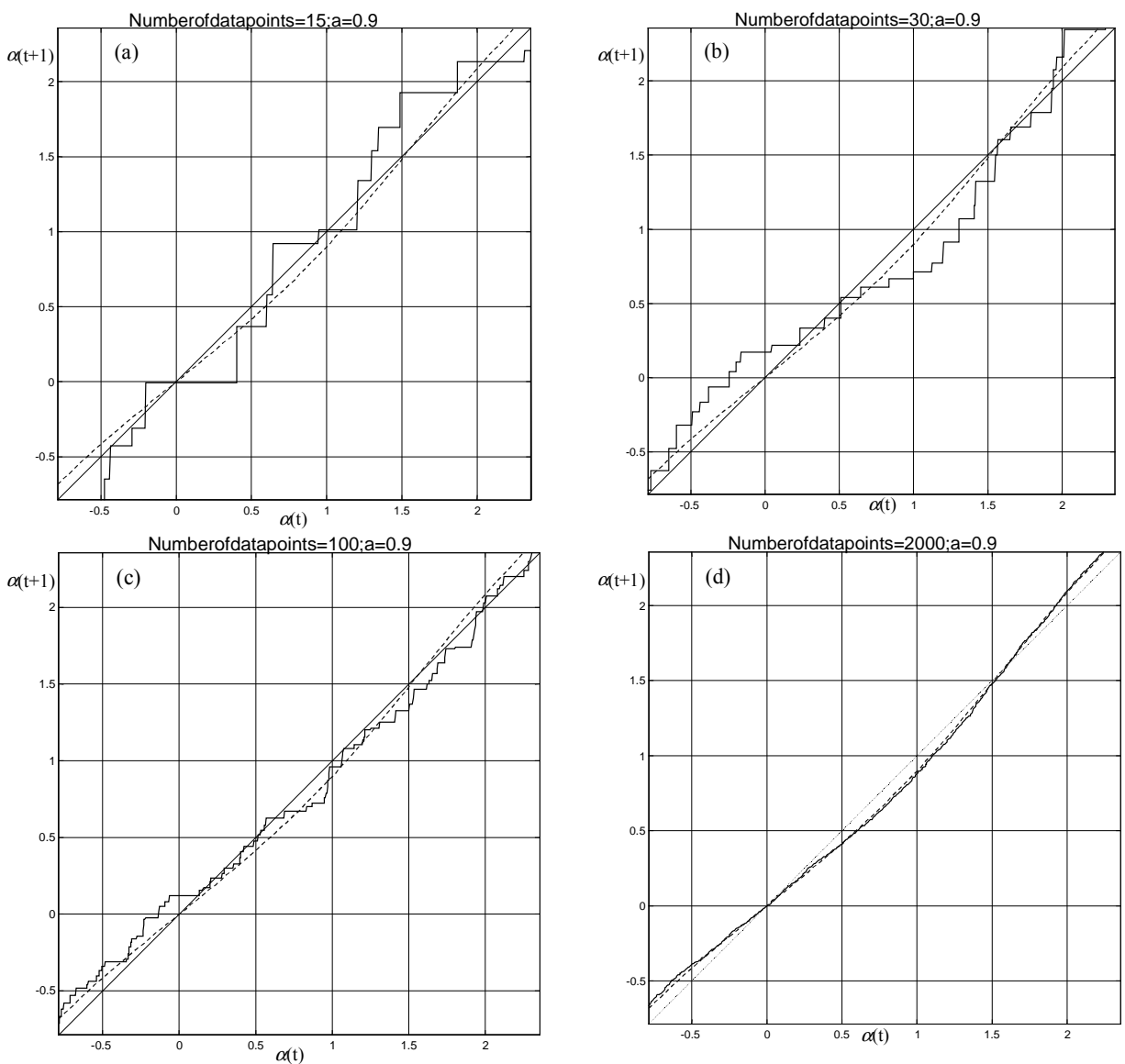

Fig.6. Recursive function $\alpha_{t+1}=f\left(\alpha_{t}\right)$ estimated from data, when $a=0.9$. The dashed line is the asymptotic function (6) computed in Section 4. (a): $N=15$; (b): $N=30$; (c): $N=100$; (d): $N=2000$.

When the number of data-point grows, the finite data-set function converges towards the asymptotic function (see Fig.5d). This validates the theoretical model 
developed in the previous section. Moreover, notice that when the number of data-point gets large, the number of equilibrium points decreases, and each step gets narrower (see e.g. Fig.5c). This explains why, when the number of data is sufficiently large, it is the common experience that the problem of local minima tends to vanish.

As a second experiment, the recursive function $\alpha_{t+1}=f\left(\alpha_{t}\right)$ has been computed for four sets of $15,30,100$ and 2000 data-points uniformly distributed within a 2dimensional ellipsoid with $a=0.9$. The results are displayed in Fig.6. The main difference in the results between the case $a=0.6$ and $a=0.9$ is that in the latter the problem of multiple equilibrium points is more severe.

The above experiments suggest that the problem of local minima for bisecting Kmeans is expected to:

- decrease when the number of data grows;

- increase when the size of the short semi-axis approaches the largest semi-axis.

In order to validate these conjectures, the bisecting K-means algorithm has been extensively tested for different values of $a(a=0.6,0.7,0.8,0.9)$ and for different sizes of the data-set ( $N$ ranging from 10 to 5000). The average dispersion of the centroids we have obtained (which is directly related to the problem of local minima) is displayed in Fig.7. In particular, for each value of $N, 20$ different data-sets have been randomly generated; for each data-set, 100 different runs of K-means have been done (starting from different initial conditions), so obtaining 100 "dispersed" centroids. The dispersion of these 100 centroids has been computed for each of the 20 data-sets, and averaged. Note that the conjectures above outlined are fully confirmed by the data: the centroids dispersion increases with $a$, and decreases with $N$.

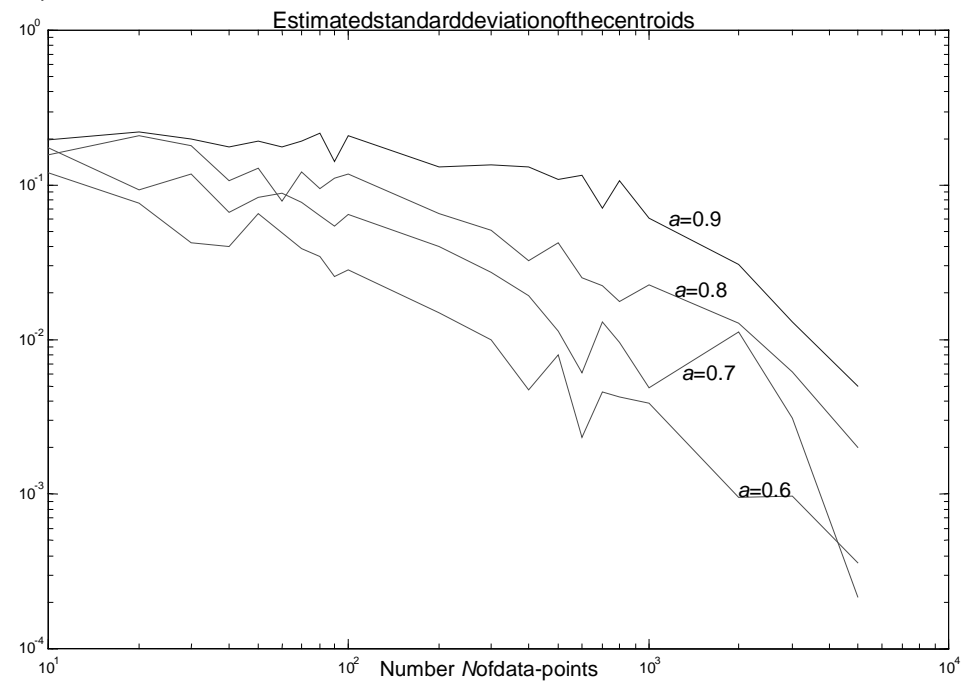

Fig.7. Average dispersion of the centroids $M_{L}$ and $M_{R}$ computed via K-means, as a function of the number of data-points. The four lines correspond to different values of $a$.

An interesting result proposed in Section 4, which must be validated, is the prediction of the number of iterations which bisecting K-means needs to converge. Recall that expression (7) is expected to hold approximately if the data set is very large. For small data-sets the convergence is expected to be faster.

To this end, the number of iterations required by K-means to converge has been experimentally estimated for different values of a in the range [0.7,0.95], using data-sets 
of size $N=20000$. The results are in Fig.8. Notice the very good fit between the predicted and the estimated results $(\gamma(N)$ used in Fig.8 to predict the number of iterations of Kmeans is $\gamma(N)=4$, which is the "rule-of-thumb value" suggested in Section 3).

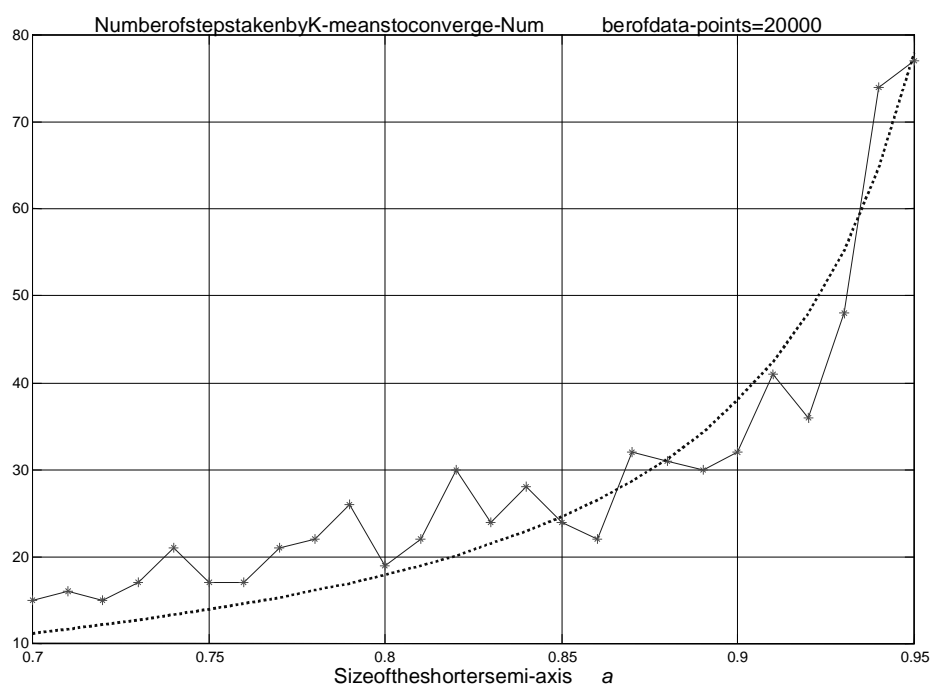

Fig.8. Estimated number of iterations required by K-means to converge, as a function of a. The dashed line is the number of iterations predicted by $(7)$, with $\gamma(N)=4$.

\section{Conclusions}

In this paper the problem of clustering a data-set is considered. Two bisecting divisive clustering techniques are considered: the K-means and the PDDP. The similarity and the differences of these two algorithms are outlined by means of a theoretical and an empirical analysis. In particular, the dynamic behavior of the recursive K-means algorithm is studied, and, under some restrictive assumptions, a closed-form model is developed.

\section{References}

[1] Anderson, T. (1954). "On estimation of parameters in latent structure analysis". Psychometrica, vol.19, pp.1-10.

[2] Berry M.W., S.T. Dumais, G.W. O'Brien (1995). "Using Linear Algebra for intelligent information retrieval". SIAM Review, vol.37, pp.573-595.

[3] Berry, M.W., Z. Drmac, E.R. Jessup (1999). "Matrices, Vector spaces, and Information Retrieval". SIAM Review, vol.41, pp.335-362.

[4] Boley, D.L. (1997). "Principal Direction Divisive Partitioning". Technical Report TR-97-056, Dept. of Computer Science, University of Minnesota, Minneapolis.

[5] Boley, D.L. (1998). "Principal Direction Divisive Partitioning". Data Mining and Knowledge Discovery, vol.2, n.4, pp. 325-344. 
[6] Boley, D.L., M. Gini, R. Gross, S. Han, K. Hastings, G. Karypis, V. Kumar, B. Mobasher, J. Moore (2000). "Partitioning-Based Clustering for Web Document Categorization”. Decision Support Systems, Vol.27, n.3, pp.329-341.

[7] Boley, D.L., M. Gini, R. Gross, S. Han, K. Hastings, G. Karypis, V. Kumar, B. Mobasher, J. Moore (2000). "Document Categorization and Query Generation on the World Wide Web Using WebACE”. AI Review, Vol.13, n.5-6, pp.365-391.

[8] Chute, C., Y. Yang (1995). "An overview of statistical methods for the classification and retrieval of patient events". Meth. Inform. Med., vol.34, pp.104110.

[9] Deerwester, S., S. Dumais, G. Furnas, R. Harshman (1990). "Indexing by latent semantic analysis". J. Amer. Soc. Inform. Sci, vol.41, pp.41-50.

[10] Forgy, E. (1965). "Cluster Analysis of Multivariate Data: Efficiency versus Interpretability of Classification”. Biometrics, pp.768-780.

[11] Golub, G.H, C.F. van Loan (1996). Matrix Computations (3rd edition). The Johns Hopkins University Press.

[12] Gose, E., R. Johnsonbaugh, S. Jost (1996). Pattern Recognition \& Image Analysis. Prentice-Hall.

[13] Jain, A.K, M.N. Murty, P.J. Flynn (1999). "Data Clustering: a Review”. ACM Computing Surveys, Vol.31, n.3, pp.264-323.

[14] Lanczos, C. (1950). "An iteration method for the solution of the eigenvalue problem of linear differential and integral operators". J. Res. Nat. Bur. Stand, vol.45, pp.255-282.

[15] LaSalle, J.P. (1986). The Stability and Control of Discrete Processes. SpringerVerlag.

[16] Selim, S.Z., M.A. Ismail (1984). "K-means-type algorithms: a generalized convergence theorem and characterization of local optimality". IEEE Trans. on Pattern Analysis and Machine Intelligence, vol.6, n.1, pp.81-86.

[17] Steinbach, M., G. Karypis, V. Kumar (2000). "A comparison of Document Clustering Techniques". Proceedings of World Text Mining Conference, KDD2000, Boston.

[18] Vidyasagar, M. (1993). Nonlinear Systems Analysis. Prentice-Hall

[19] Wang, J.Z., G. Wiederhold, O. Firschein, S.X. Wei (1997). "Content-based image indexing and searching using Daubechies' wavelets". Int. J. Digit. Library, vol.1, pp.311-328. 